months after the injection. After a single small dose of cells, anti-Rh can usually not be detected serologically, but its presence is indicated by the diminished survival of a second dose of Rh-positive red cells, followed by the appearance of readily detectable antibody.

There is evidence that the minimum effective dose for primary $\mathrm{Rh}$ immunization is less than $0.1 \mathrm{ml}$ of red cells; if so, more than $20 \%$ of Rh-negative women receive an adequate primary stimulus during a pregnancy with an Rh-positive foetus.

SUPPRESSION OF PRIMARY RH IMMUNIZATION

Recent work suggests that with doses of red cells between 13 and $200 \mathrm{ml}, 25 \mu \mathrm{g}$ of anti-Rh per ml of cells is effective in suppressing immunization (Dr W. Pollack, personal communication). For $1 \mathrm{ml}$ of red cells, $15 \mu \mathrm{g}$ seems to be only partially effective and $75 \mu \mathrm{g}$ to be completely effective; intermediate doses have not been tested. Only about $2 \%$ of women have a 'TPH' of more than $1 \mathrm{ml}$ of red cells.

There is no decisive evidence to show that either IgG or IgM anti-Rh, when given with $\mathrm{Rh}$-positive red cells, auguments the immune response.

Under conditions which have been shown to result in suppression of $\mathrm{Rh}$ immunization, only about $5 \%$ of the $\mathrm{Rh}$ antigen sites on the red cell are covered by antibody. This finding suggests the possibility that anti-Rh may exert its suppressing effect simply by causing the destruction of red cells, and so of antigen, rather than by 'masking' of antigen sites. On the other hand, ABO incompatibility, which also results in destruction of antigen, protects only partially against $\mathrm{Rh}$ immunization, supporting the idea that the role of 'passive' antibody is specific.

\section{Liquor Examination in the Management of RH Isoimmunization}

W. WALKER (Royal Victoria Infirmary Newcastle-upon-Tyne)

Liquor examination, particularly for the presence of bile pigment, is widely used in the management of pregnancies complicated by $\mathrm{Rh}$-isoimmunization. Neither the source nor route of entry of this pigment is known but high liquor levels are often associated with severe haemolytic disease of the newborn, nonetheless, application in management is essentially empirical. The purpose in forecasting severity is that premature induction or intrauterine transfusion may be carried out to try and prevent intrauterine death.

Intrauterine death is relatively rare and both forms of treatment are relatively dangerous so that accuracy of prediction is essential and risks of amniocentesis must be minimal.

Various different methods of quantitating bilirubin in liquor amnii are employed. No method is significantly superior to the other and errors in prediction are rarely due to errors in detecting bilirubin, provided precautions are taken in the collection and preparation of the specimen.

A major source of error is in assessing gestation accurately, for the bilirubin value is closely related to this. It may also depend on variations in the value of other constituents of liquor, and some have been estimated in order to try and improve its predictive value.

The pattern of liquor bilirubin in normal pregnancy and those complicated by $\mathrm{Rh}$ isoimmunization will be considered, and mention will be made of other constituents. Particular reference will be made to cases in which wrong forecasts have been made.

\section{Abnormalities of the Lower Spine and Spinal Cord in Manx Cats}

B. E. TOMLINSON (Newcastle-upon-Tyne)

The 'tailless' Manx cat occurs in two forms, one with a very short tail and known on the Isle of Man as a stumpy, the other with no apparent tail (a rumpy). Tailless Manx cats are commonly described as having a characteristic rabbity or hopping gait, though many actually show severe physical disabilities, particularly incontinence of urine and faeces and hind-limb paralysis.

The lower spine and spinal cord of 13 Manx cats have been examined.

Three possessed short tails (stumpies), and all were said to be free from disability. Grossly the spinal cords and lumbosacral vertebrae were normal the only abnormality being the reduced number (four to six) of caudal vertebrae. Histologically the spinal cords were normal in two, but one showed severe hydromyelia of the sacrococcygeal segments.

Nine of 10 rumpies showed other abnormalities in addition to absent or rudimentary caudal vertebrae. Five of these nine showed sacral dysgenesis, and all nine showed spinal cord abnormalities. In three the filum terminale and in five the lower sacral or coccygeal cord was attached to the dura beyond the sacrum and covered only by subcutaeous tissue and fur.
Traction on the rump fur pulled down the lower cord. Two animals had 'mening음 ocoele'-like cysts, four showed intradura fat masses (lipomas), one had a split lowe $\overline{\bar{\sigma}}$ cord (diastematomyelia), and two lumbo sacral degenerative cord lesions wit|f cavitation. Only one rumpy showed nछ apparent spinal cord lesion.

\section{Leucocytic Infiltration of the Placenta: Clinico-pathological Study}

H. FOX AND F. A. LANGLEY (Manchester) Leucocytic infiltration of the placenta oR membranes was found in $24.4 \%$ of 8 series of 1,000 placentas. The only clinicain factor significantly related to placentaf leucocytic infiltration was prolongeo

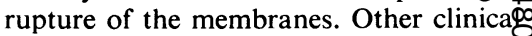
factors such as premature onset of labour artificial rupture of the membranes, proo longed pregnancy, pre-eclamptic toxaemia? and prolonged labour showed an apparent relationship to placental leucocytic infile tration but this was due to the high proportion of cases in these groups in whicke prolonged membrane rupture occurred if this factor was taken into account ther was no significant relationship with these clinical factors. Placentas from hypoxis foetuses were more commonly infiltrate by leucocytes than were those in whicl there was no evidence of hypoxia. Thi was related to the high incidence of foeta hypoxia in cases in which there was pro을 longed membrane rupture, and analysi showed that, if this was taken into accoun there was no significant association between the placental lesion and foeta市 hypoxia. Similar findings were found if $6.6 \%$ of cases in which leucocytic infilo tration of the cord was present.

It is concluded that leucocytic infiltra@ tion of the placenta, membranes, or cord is indicative of bacterial infection and i오 not related to foetal hypoxia.

Quantitation of Tissue Components in the
Bronchi in Chronic Bronchitis and Emphy
sema
G. R. MASSARElla (Royal Postgraduato Medical School, London)

Bronchi from lungs obtained at necrops from patients known to have had obstruc tive airways disease and from a controt group of 16 patients were examined. The mean Reid index for the groups was 0.52 and 0.36 respectively. The area of the bronchial lumen, mucous glands, cartilage $\overrightarrow{\mathrm{P}}$ and connective tissue (all tissue compo 
nents of the bronchial wall apart from the mucous glands and cartilage) was measured in numerous bronchi from each lung. This was done by tracing each component at a suitable magnification on a Projectina microscope, and then measuring the traced area on an image analyser. The bronchi were then divided into four groups of the following external diameters: (1) 3-3.99 mm, (2) $4-4.99 \mathrm{~mm}$, (3) 5-5.99 mm, (4) 6-6.99 mm. Significant differences $(P<0.01)$ were found in the mean areas of the mucous glands in the control and obstructed patients in groups (3) and (4), and of the mean area of cartilage in groups (1) and (2). The latter probably indicates that dividing the bronchi into groups by size rather than generation leads to comparison of different generations of bronchi, and the implication of this on luminal size will be discussed.

\section{Lung Cancer in Young Adults}

A. KENNEDY (Sheffield Royal Infirmary and University of Sheffield)

This is an account of 36 cases in which the diagnosis of lung cancer was established histologically before the age of $\mathbf{4 0}$ years. The dominant feature of the pathology is that two-thirds of the tumours were oat cell carcinomas, and that all but one of the patients under 33 had oat-cell tumours. Only three patients were known to be non-smokers; four tumours arose in lung scars but no other aetiological factor was evident. Of the 10 women in the series three were pregnant at the time of appearance of the tumour or its metastases. There was a high death rate which is attributable to the large proportion of oatcell tumours. The only long-time survivors are patients who had tumours other than of the oat-cell type and who were seen early enough for radical surgery. One of the patients had a pulmonary blastoma but she is alive 14 years after pneumonectomy and 11 years after the appearance of metastases.

Glandular Metaplasia and Mucin Production in Transitional Cell Carcinomas of Bladder

A. M. WARD (Department of Pathology, University of Sheffield)

Three hundred and forty cases of transitional cell carcinoma of bladder seen in the Urological Unit, United Sheffield Hospitals, between 1967 and 1970 are reviewed. Of these 340 cases, $25-30 \%$ show evidence of mucin production, and a similar, but not necessarily identical percentage show some form of adenomatous metaplasia or glandular configuration. Mucin production and adenomatous metaplasia do not affect the grading of any particular tumour, and are seen with equal frequency in low, intermediate, and high grade tumours. There is no relationship to infection or prior instrumentation, and the changes have no apparent prognostic significance. Tumours showing mucin production and adenomatous metaplasia emphasize the metaplastic potential of the urothelium; they will behave biologically as transitional cell carcinomas, and should be treated as such.

Ultrastructural Evidence of Secretion in Hormonally Active Oat-cell Carcinomas: Origin of One Such Tumour in the Pancreas

B. CORRIN, E. D. GILBY, MARY MCMILLAN, J. PATRICK, AND H. SPENCER (St. Thomas's and Lewisham Hospitals, London)

An electron-microscopical study of 48 lung tumours identified characteristic cytoplasmic granules in oat-cell carcinomas and bronchial carcinoid tumours, but not in large cell anaplastic carcinomas. The granules were similar to those found in the cells of many endocrine glands. Sparse cells containing such /granules were also identified in normal bronchial epithelium. These resembled intestinal Kultschitzky cells and are believed to represent the cell of origin of both the bronchial carcinoid and oat cell carcinoma (Bensch, Corrin, Pariente, and Spencer, 1968). If this is so, why do oat-cell carcinomas not arise in the gastrointestinal tract, and what is the fine structure of oat-cell carcinomas associated with inappropriate endocrine activity?

Two cases of oat-cell carcinoma associated with ectopic ACTH production are presented, one arising in the lung, the other in the pancreas. Electron microscopy of the pulmonary neoplasm showed that the cytoplasmic granules were more plentiful than in non-secretory tumours, supporting the suggestion that they represent secretory activity, even in those tumours in which hormonal activity is not clinically manifest, and that all oat-cell carcinomas may be regarded as latent endocrine neoplasms. In the second case necropsy showed that the tumour was limited to the tail of the pancreas, local lymph nodes, and the liver. Detailed examination of the lungs disclosed no new growth although histologically the tumour was a typical oat cell carcinoma. McKeown (1952) has reported two oat-cell carci- nomas arising in the oesophagus, and it $\stackrel{0}{\overrightarrow{7}}$ would appear that although an extrapul- 음 monary origin is rare these tumours are not restricted to the lung.

References

Bensch, K. G., Corrin, B., Pariente, R., and Spencer, H. (1968). Oat-cell carcinoma of the lung. Its origin and relationship to bronchial carcinoid. Cancer. (Philad), 22, هి 1163-1172.

McKeown, Florence (1952). Oat cell carcinoma of the oesophagus. J. Path. Bact., 64, 889-891.

\section{Asbestos in Tumours}

C. GOLD (M.R.C. Pneumoconiosis Unit, Penarth, Glamorgan)

Epidemiological evidence suggests that the four main types of asbestos, although $\frac{\vec{A}}{\mathrm{a}}$ all carcinogenic in animals, do not appear to be equally implicated in the causation $\stackrel{\circ}{\circ}$ of human tumours. Furthermore, in the three types of asbestos-related malignant 0 lesions there are obvious site and be- havioural differences between lung car- $\vec{c}$ cinoma and mesotheliomas of pleura or peritoneum. Variable factors such as type and source of asbestos, fibre characteris- $c$ tics, site of deposition, cellular, chemical $\perp$ and physical reactions, and total dose of dust may be all, or in part, responsible. Thus it would seem important to establish among other things if human neoplasia are $\overline{0}$ related only to certain types of asbestos or if there is a dose-response relationship.

Bronchial carcinomas tend to have $a \underset{F}{F}$ high lung asbestos content while a pleural 윽 mesothelioma lung can contain relatively little asbestos. On the other hand, in peritoneal mesotheliomas the lungs may have an intermediate value or sometimes a considerable amount of asbestos in them without having a pleural or intrapulmonary $\exists$ tumour. With these apparent paradoxes a correlative investigation of both the dust 0 and the histopathological changes seem justified. This approach is illustrated by results in selected referred human lung $D$ cancers and mesotheliomas.

The simple $\mathrm{KOH}$ extraction method yields a concentrated dust residue suitable $\sigma$ for quantitative and morphological study $\mathrm{N}$ with the light microscope and useful for $\stackrel{N}{N}$ the identification and typing of asbestos $\omega$ in the electron microscope. In experimental animal work with pure exposures 0 to the individual types of asbestos very $\frac{\bar{D}}{\mathscr{}}$ high fibre counts can be obtained and $\stackrel{?}{?}$ virtually pure dust reclaimed.

Experimental Tumour Induction in the Rat $\frac{\vec{D}}{\mathbb{D}}$ Nervous System by $\mathbf{N}$-Ethyl N-Nitrosourea $\frac{O}{\mathbb{D}}$ E. L. JONES, W. THOMAS SMITH, AND C. E. ¿ SEARLE (Departments of Pathology and 